\title{
Characterization of IS900 loci in Mycobacterium avium subsp. paratuberculosis and development of multiplex PCR typing
}

\author{
Tim J. Bull, ${ }^{1}$ John Hermon-Taylor, ${ }^{1}$ Ivo Pavlik, ${ }^{2}$ Fouad El-Zaatari ${ }^{3}$ \\ and Mark Tizard ${ }^{4}$
}

Author for correspondence: Tim J. Bull. Tel: +44 181725 5580. Fax: +44 1817253594.

e-mail: tim.bull@sghms.ac.uk

1 Department of Surgery, St George's Hospital Medical School, Cranmer Terrace, London, UK

2 Veterinary Research Institute, Brno, Czech Republic

3 Baylor College of Medicine, Houston, TX, USA

4 CSIRO Division of Animal Health, PO Box 24, Geelong, VIC 3220, Australia
Mycobacterium avium subsp. paratuberculosis is a pathogen that causes chronic inflammation of the intestine in many animals, including primates, and is implicated in Crohn's disease in humans. It differs from other members of the $M$. avium complex in having 14-18 copies of IS900 inserted into conserved loci in its genome. In the present study, genomic DNA flanking 14 of these insertions was characterized and homologues in the Mycobacterium tuberculosis and $M$. avium subsp. avium genomes were identified. These included regions encoding a sigma factor (sigل) at locus 3, a nitrate reductase (nirA) at locus 4, a transcription regulator (tetR) and polyketide synthase at locus 6, and a 6-0-methylguanine methyltransferase at locus 9. In addition, locus numbers were assigned to 9 of 15 RFLP bands previously described. IS900 insertion at 7 of the 14 characterized loci was into the RBS of a gene substituting an RBS encoded by IS900 sited two bases closer to the initiation codon. IS900 insertion at five loci interrupted an ORF at the target site, one of which encoded a homologue of the immunodominant mycobacterial DesA1 protein. Eleven of eighty-one $M$. avium subsp. paratuberculosis isolates lacked the insertion site at locus 6 together with flanking genomic DNA. This region was also absent from seven reference strains of $M$. avium subsp. avium, from one $M$. avium subsp. silvaticum and from six other mycobacterial species. A multiplex PCR of IS900 loci (MPIL) typing method was developed which was able to discriminate 10 different types of $M$. avium subsp. paratuberculosis from the panel of 81 isolates with consistent differences between those of bovine and ovine origin. Nine MPIL types corresponded with a single PstI/BstEII RFLP type, suggesting that this method may be applicable to typing of $M$. avium subsp. paratuberculosis directly from a sample without the need for culture. The remaining MPIL type corresponded with seven PstI/BstEII RFLP types. Further resolution of these may come from sequencing the remaining four uncharacterized IS900 loci.

Keywords: multiplex, IS900, typing, Mycobacterium avium subsp. paratuberculosis

\section{INTRODUCTION}

Mycobacterium avium subsp. avium is widely distributed in the environment and is harboured in the intestines of healthy animals and humans (Portaels et

Abbreviation: MPIL, multiplex PCR of IS900 loci.

The GenBank accession numbers for the sequences reported in this paper are AJ011838, AJ250015-AJ250023 and AJ251434-AJ251437. al., 1988; Kallinowski et al., 1998). It rarely causes disease in immunocompetant hosts. M. avium subsp. paratuberculosis, which is very closely related to other M. avium subspecies, is a specific pathogen able to cause chronic inflammation of the intestine in ruminants, monogastrics such as dogs and pigs, and in subhuman primates (Larsen et al., 1971; McClure et al., 1987; Chiodini, 1989; Cocito et al., 1994). Our understanding of the genetic basis for this pathogenicity in $M$. avium subsp. paratuberculosis will only begin to ac- 
celerate when the whole genome sequence of at least one strain is available for scrutiny. At the present state of our knowledge however, one of the principal differences between these pathogens and other members of the $M$. avium complex is the presence within $M$. avium subsp. paratuberculosis of 14-18 copies of the insertion element IS900 (McFadden et al., 1987; Green et al., 1989). The presence of insertion elements has been associated with the pathogenic phenotype in other organisms (Bartlett \& Silverman, 1989). These elements have also been used for typing culturable isolates of $M$. avium subsp. paratuberculosis (McFadden et al., 1987; Whipple et al., 1990; Moss et al., 1991; Pavlik et al., 1995, 1999; Bauerfeind et al., 1996; Collins et al., 1997; Whittington et al., 1998; Moreira et al., 1999). This approach to the molecular typing of M. avium subsp. paratuberculosis is, however, limited by the very slowgrowing nature of most strains and lack of growth of others, particularly from sheep, in conventional culture.

IS900, which is unique for M. avium subsp. paratuberculosis, encodes a 399 aa putative transposase, p43, on one strand (Tizard et al., 1992) and a predicted protein, Hed, of unknown function on the opposite strand (Doran et al., 1994). IS900 inserts in one direction into a consensus target sequence at highly conserved loci within the M. avium subsp. paratuberculosis genome. Previous studies have suggested that the arrangement at some loci is one in which IS900 inserts between the RBS and start codon of the target gene such that the hed ORF, which contains its own start codon within IS900, comes under the control of the upstream host promoter (Tizard et al., 1992; Doran et al., 1997). In the present paper, we have used the term 'hed orientation' to describe the situation in which the predicted direction of genomic transcription at the target locus is in the same direction as hed. The use of the term ' $p 43$ orientation' follows the same principles. Our own recent studies (T. J. Bull, unpublished) have identified an endogenous promoter within IS900 itself controlling the expression of $\mathrm{p} 43$. This may also influence the expression of host genes immediately downstream, as has been described for insertion elements in other organisms (Kallastu et al., 1998; Ziebuhr et al., 1999). Much, however, remains to be learned about the detailed organization of IS900 insertions and how they may affect the phenotype of these pathogens.

In the present study we have sequenced flanking genomic regions for 14 IS900 loci present in M. avium subsp. paratuberculosis. In addition, we have used the sequence information to develop a multiplex PCR typing method applicable to unculturable strains of $M$. avium subsp. paratuberculosis which reports the presence or absence of the IS900 at each locus.

\section{METHODS}

IS900 clone selection, sequencing and BLAST analysis. Two total genomic libraries of $M$. avium subsp. paratuberculosis DNA (strain 0138 'Linda' of human origin and 0142 of primate origin; McClure et al., 1987) were cloned into
pcDNAII expression vectors (Invitrogen) and screened for the presence of IS900 using PMB22/S12 or specific PCR primers as previously described (El-Zaatari et al., 1994; Pavlik et al., 1999). Fourteen distinct clones were identified by differences in Pst I and XhoI restriction digest patterns. These were sequenced with IS900 primers (IS9R: ACGCTGTCTACCACCCCGCA; and IS9L: ACCACATGAAAGCCATACCC) using standard GC-rich sequencing protocols (Applied Biosystems). Sequences were then verified by resequencing in the complementary direction (towards IS900) using vector primers (SP6 and T7) and the following Loc primers designed to be specific for each separate locus: Loc1R, CCACCCTCGTTCCGGTCGTG; Loc1L, CCCGTGACAAGGCCGAAGA; Loc2R, GTCGACCATCGCTCTTCCCT; Loc2L, ATCGGTGTCGAGGACATTCC; Loc3R, CGCGGACAGACACAGGTAGG; Loc3L, ACAATTCGGCGATCGTCTCG; Loc4R, TATTCGGGGTTGTTCAGGGA; Loc4L, CCGCAAGTAGTGCACTATGG; Loc5R, GACAATCTGCCGTCGTATCA; Loc5L, TCCCGGTAGAAGATCATGTG; Loc6R, CTACGGTGGCCAGCGTTTCT; Loc6L, GGTTGGAGACAACCTCGTTC; Loc7R, TCCTCGACGTGCTCGTGGAA; Loc7L， GTGACCGGAATCATCTTGCTG; Loc8R, TGGGCCTGAGGTCAGAACCA; Loc8L, GAAGACCACCTCTACCTCAC; Loc9R, TGAGCTCGCCGGCGAAATA; Loc9L, CGGCCCTGGCGTTCCTATG; Loc10R, AGCTGCCGGGAGTTGATCT; Loc10L, TCACGGCTCACCGCACGCT; Loc11R, CGTGACGAACGACATGTGTT; Loc11L, GCTCGAGACATTTAGCCCAC; Loc12R, CAGGCACGGAACACAGTTCG; Loc12L, TGTTCGGCTACGGCATACTG; Loc13R, CGGCCCGCTTGATCCATTTG; Loc13L, CAATGGATGGTCGTCACCTG; Loc14R, TCCTCGACGTGCTCGTGGAA; Loc14L, CGTTCTGAGCATCCATCGACG

BLASTX alignments of DNA sequences flanking each locus were made against the M. avium subsp. avium (TIGR strain 104) genome. TBLASTN analyses in GenBank of predicted ORFs 'upstream' and 'downstream' from each IS900 locus were also made. Genes showing $>30 \%$ identity were assigned putative functions. The direction of transcription of these flanking genes was determined by comparison with homologues in Mycobacterium tuberculosis, Mycobacterium leprae and Streptomyces coelicolor (Cole et al., 1998; Eiglmeier et al., 1993; Redenbach et al., 1996).

M. avium subsp. paratuberculosis strains, DNA preparation and RFLP. A panel of 81 strains of $M$. avium subsp. paratuberculosis including representatives of 17 Pst I/Bst EII RFLP profiles were investigated (Table 1). Total DNA was extracted from $M$. avium subsp. paratuberculosis isolates grown on Herrold's egg-yolk medium (USDA, 1974). Briefly, one loop of colonies was emulsified in $1 \mathrm{ml}$ sterile PBS (Dulbecco's PBS; Sigma), centrifuged (13000 $\mathrm{g}$ for $5 \mathrm{~min}$ ) and the pellet washed in $1 \mathrm{ml} \mathrm{TE}\left(10^{-4} \mathrm{MEDTA}, 10^{-2} \mathrm{M}\right.$ Tris $/ \mathrm{HCl}$ $\mathrm{pH} 8 \cdot 0)$. The pellet was emulsified in $0.6 \mathrm{ml} \mathrm{TE}$, heated at $80^{\circ} \mathrm{C}$ for $20 \mathrm{~min}$, cooled and treated with lysozyme (Sigma; $1 \mathrm{mg} \mathrm{ml}^{-1}$ final concentration) for $90 \mathrm{~min}$ at $37^{\circ} \mathrm{C}$ followed by proteinase $\mathrm{K}$ (Sigma; $150 \mu \mathrm{g} \mathrm{ml}{ }^{-1}$ final concentration)/SDS (Sigma; $1 \%$, w/v, final concentration) for $30 \mathrm{~min}$ at $65^{\circ} \mathrm{C}$. The concentration was then adjusted to $0.5 \mathrm{M} \mathrm{NaCl}, 1 \%$ (w/v) CTAB [stock: $1 \mathrm{~g}$ cetyltrimethylammonium bromide (BDH), $\left.0.4 \mathrm{~g} \mathrm{NaCl}, 10 \mathrm{ml} \mathrm{dH}_{2} \mathrm{O}\right]$ and incubated for $30 \mathrm{~min}$ at $65^{\circ} \mathrm{C}$. DNA was then extracted with 1 vol. chloroform/isoamyl alcohol $(24: 1)$ and precipitated with 1 vol. 2-propanol (BDH) for $1 \mathrm{~h}$ at $-70^{\circ} \mathrm{C}$, centrifuged $(13000 \mathrm{~g}$ for $15 \mathrm{~min})$, washed in $70 \%$ ethanol, dried and resuspended in TE. RFLP profiles were produced on DNA digested with BstEII or PstI (Roche) and probed with an IS900 
Table 1. M. avium subsp. paratuberculosis type strains used in this study

\begin{tabular}{|c|c|c|c|c|}
\hline $\begin{array}{l}\text { RFLP* } \\
(\text { PstI })\end{array}$ & $\begin{array}{l}\text { RFLP } \\
(\text { BstEII })\end{array}$ & $\begin{array}{l}\text { No. of } \\
\text { strains }\end{array}$ & Strain ID (host) $\dagger$ & Origin \\
\hline \multirow[t]{7}{*}{ B } & $\mathrm{C} 1$ & 32 & $\begin{array}{l}\text { Tb429/94(B), Tb411/94(B), 522/94(B), } \\
\text { Tb1636/95(B), Tb149/96(B) }\end{array}$ & Sweden \\
\hline & & & $\begin{array}{l}\mathrm{P} 2525 / 96(\mathrm{~B}), \mathrm{P} 1835 / 6(\mathrm{~B}), \mathrm{P} 2294 / 6(\mathrm{~B}) \\
\mathrm{P} 2514 / 6(\mathrm{~B}), 7848(\mathrm{~B}), 7851(\mathrm{~B})\end{array}$ & Czech Republic \\
\hline & & & $\begin{array}{l}7862(\mathrm{D}), 8496(\mathrm{D}), 7509(\mathrm{~B}), \mathrm{P} 2142 / 5(\mathrm{~B}), 6533(\mathrm{~B}) \\
8543(\mathrm{~B}), 9882(\mathrm{~B}) 4600(\mathrm{~B}), 4604(\mathrm{~B}), 9237(\mathrm{~B})\end{array}$ & Slovak Republic \\
\hline & & & $\operatorname{Jeltema}(\mathrm{H}), \operatorname{Van} \operatorname{Veen}(\mathrm{H})$ & The Netherlands \\
\hline & & & $\mathrm{P} 2570 / 93(\mathrm{G})$ & Norway \\
\hline & & & $\operatorname{VIAS}(\mathrm{H})$ & Australia \\
\hline & & & $\begin{array}{l}\operatorname{SD} 39 \mathrm{~T}(\mathrm{~B}), 105 \mathrm{~T}(\mathrm{~B}), \mathrm{SD} 620(\mathrm{~B}), 95 / 28(\mathrm{~B}) \\
\mathrm{DM} 645(\mathrm{~B}), 95 / 371 \mathrm{a}(\mathrm{D}), 95 / 371 \mathrm{~b}(\mathrm{D})\end{array}$ & Argentina \\
\hline B & $\mathrm{C} 1$ & 11 & $\begin{array}{l}9456 \mathrm{HE}(\mathrm{B}), 9478 \mathrm{HE}(\mathrm{B}), 9393 \mathrm{HE}(\mathrm{B}), 9562 \mathrm{HE}(\mathrm{B}), \\
\text { 9392HE(B), } 9441 \mathrm{HE}(\mathrm{B}), 8376(\mathrm{~B}), 9222(\mathrm{~B}) \\
9235(\mathrm{~B}), 4604(\mathrm{~B}), 10344(\mathrm{~B})\end{array}$ & Slovak Republic \\
\hline B & $\mathrm{C} 3$ & 3 & $12 \cdot 970677(\mathrm{~B}), \mathrm{M} 212(\mathrm{~B}), 1091(\mathrm{~B})$ & USA \\
\hline \multirow[t]{3}{*}{ B } & $\mathrm{C} 5$ & 6 & $\operatorname{Ben}(H)$, Dominic $(H)$, Linda $(H)$, Primate & USA \\
\hline & & & $\mathrm{P} 2142 / 5(\mathrm{~B})$ & Denmark \\
\hline & & & P2157/6(B) & Czech Republic \\
\hline B & C9 & 1 & $9409(\mathrm{~B})$ & Czech Republic \\
\hline \multirow[t]{2}{*}{ A } & $\mathrm{C} 10$ & 3 & 4/552(B), 10/965Fryba $(\mathrm{H})$ & Czech Republic \\
\hline & & & $7844(\mathrm{~B})$ & Slovak Republic \\
\hline K & C11 & 2 & $1036(\mathrm{~B}), 1113(\mathrm{~B})$ & USA \\
\hline A & C12 & 1 & $\mathrm{P} 220 / 4(\mathrm{~B})$ & USA \\
\hline $\mathrm{D}$ & C12 & 4 & P3015/6(B), 8355(B), 7503(B), 4062-Michaela $(\mathrm{H})$ & Czech Republic \\
\hline $\mathrm{L}$ & C13 & 3 & $\mathrm{P} 769(\mathrm{~B}), 5075(\mathrm{~B}), 5080(\mathrm{~B})$ & Slovak Republic \\
\hline B & C15 & 1 & $1030(\mathrm{~B})$ & USA \\
\hline B & C16 & 1 & $\mathrm{R} 8$ & UK \\
\hline B & C17 & 7 & R7, R186, F14, JD4, F54, M101, M103 & UK \\
\hline B & C18 & 1 & 960735 & USA \\
\hline B & C19 & 1 & $93 / 433(\mathrm{~B})$ & Argentina \\
\hline $\mathrm{F}$ & I1 & 2 & $575 \mathrm{a}(\mathrm{S}), 506 \mathrm{c}(\mathrm{S})$ & Australia \\
\hline $\mathrm{C}$ & $\mathrm{S} 1$ & 2 & $6760 \mathrm{~b}(\mathrm{~S}), 6758 \mathrm{~b}(\mathrm{~S})$ & Australia \\
\hline
\end{tabular}

* RFLP types taken from Pavlik et al. (1999).

$\dagger(B)$, bovine; $(\mathrm{H})$, human; (S), sheep; $(\mathrm{G})$, goat; (D), deer.

probe using standardized procedures, as previously described (Pavlik et al., 1999). Profiles were analysed on GelCompar software (Biosystematica).

Multiplex PCR of IS900 loci (MPIL). PCR reaction master mixes were made of the following combinations of LocR primers + IS9L primer, or LocL primers + IS9R primer, to test for the presence of an IS900 element at individual loci in total M. avium subsp. paratuberculosis DNA. The expected sizes of each PCR product (bp) are given in parentheses. Multiplex 9R1: IS9L, Loc13R(203), Loc9R(265), Loc5R (340), Loc10R(386), Loc7R(409), Loc2R(543), Loc1R(765), Loc12R(893), Loc6R(1044); multiplex 5R2: IS9L, Loc11R (102), Loc8R(143), Loc14R(285), Loc3R(509), Loc4R(693); multiplex 4L1: IS9R, Loc9L(147), Loc2L(224), Loc6L(267), Loc3L(560); multiplex 4L2: IS9R, Loc1L(207), Loc11L(245), Loc12L(326), Loc7L(466); multiplex 3L3: IS9R, Loc5L(185), Loc10L(206), Loc4L(244); multiplex 3L4: IS9R, Loc14L (150), Loc8L(226), Loc13L(263). Loc primers were designed to locate at distances increasing by at least $20 \mathrm{bp}$ from their neighbouring IS900 element so that the amplification products from the multiplex PCR could be easily seen on a single electrophoresis gel.

Correlation of RFLP bands with IS900 loci. To assign each BstEII RFLP band to a sequenced IS900 locus, 13 individual portions of gel each containing one RFLP band, and an additional portion containing two RFLP bands which could not be separated, were excised from a $M$. avium subsp. paratuberculosis BstEII RFLP C1 profile. The DNA was purified from each portion of gel (Qiagen gel extraction kit 28706) and amplified using multiplex PCR 9R 1 and multiplex PCR 5R2 reaction mixes. The strongest PCR amplifications of the predicted size were then matched to corresponding RFLP bands and could then be assigned specific IS900 loci (Fig. 6).

Insertion locus PCR. Strains of $M$. avium subsp. paratuberculosis that gave multiplex PCR profiles which did not amplify all 14 loci (left and right) were further tested using primer pairs specific for each IS900 locus in separate PCR reactions. 'Filled' loci gave large PCR products containing IS900 plus the flanking DNA. 'Unfilled' loci lacking IS900 
gave products which were 1451 bp shorter. The expected size of 'unfilled' products for each locus were as follows: Loc 1, 830 bp; Loc 2, 687 bp; Loc 3, 844 bp; Loc 4, 796 bp; Loc 5, 387 bp; Loc 6, 1171 bp; Loc 7, 789 bp; Loc 8, 230 bp; Loc 9, $281 \mathrm{bp}$; Loc 10, 462 bp; Loc 11, 159 bp; Loc 12, 1077 bp; Loc 13, $324 \mathrm{bp}$; Loc 14, $411 \mathrm{bp}$. Loci which gave no products with any of the LocR or LocL primers, but did give a product with IS9R or IS9L primers were further investigated for possible genomic rearrangements, using combinations of various LocR and LocL primer pairs from other loci. Multiplex PCR data from each of the panel of $M$. avium subsp. paratuberculosis strains was combined with insertion locus PCR data to give a profile which was assigned an MPIL type for comparison with the RFLP type.

The presence of locus 6 in M. avium subsp. paratuberculosis and $\boldsymbol{M}$. avium subsp. avium. Eleven of the eighty-one strains of $M$. avium subsp. paratuberculosis did not amplify products with any locus-6-specific primers. To investigate this further, PCR for the presence of genes previously located on either side of the locus (tetR and $p k s$ ) using the primers tetR.F: TTGGGCCTTGACTCCATGAC; tetR.R: AGCAGAAGGAACGCAACCGT; $p k s . F$ : GGAGTATGGAACCATCGGTG; pks.R: TGATGTAACGGGCGTGCAAG; or locus-6spanning primers tetR.F + pks.R, was performed on total DNA samples from the complete panel of 81 M. avium subsp. paratuberculosis strains, as well as the following additional mycobacterial strains: $M$. avium subsp. avium serotype 1 (ATCC 35717), M. avium subsp. avium serotype 2 (NCTC 8551), M. avium subsp. avium serotype 2 (NCTC 8553), $M$. avium subsp. avium serotype 2 (NCTC $8559=$ ATCC 19421), $M$. avium subsp. avium serotype 4 (strain MAAnewc4), $M$. avium subsp. avium serotype 8 (strain MAAmjg16), M. avium subsp. avium serotype 2 (ATCC 25291), M. avium subsp. silvaticum (wood pigeon strain 012), Mycobacterium intracellulare (NCTC 10682), Mycobacterium malmoense (NCTC 11298), M. tuberculosis H37Rv (NCTC 7416), Мycobacterium phlei (ATCC 11756), Mycobacterium fortuitum (NCTC 10394), Mycobacterium smegmatis (NCTC 10265).

PCR reaction conditions. All PCRs used the following reagents and reaction conditions. The PCR mix consisted of a $50 \mu \mathrm{l}$ reaction volume containing a final concentration of $20 \mu \mathrm{M}$ of each Loc primer, $100 \mu \mathrm{M}$ of each IS900 primer, $10 \%$ (v/v) DMSO, 1.5 mM MgCl, 100 mM dNTP, 2 U Taq polymerase (Promega) in $1 \times$ reaction buffer (Promega). Reactions were cycled as follows: $94^{\circ} \mathrm{C}$ for $3 \mathrm{~min}\left(1\right.$ cycle); $94^{\circ} \mathrm{C}$ for $30 \mathrm{~s}$, $60{ }^{\circ} \mathrm{C}$ for $30 \mathrm{~s}, 72^{\circ} \mathrm{C}$ for $2.5 \mathrm{~min}(40$ cycles $) ; 72^{\circ} \mathrm{C}$ for $5 \mathrm{~min}(1$ cycle). PCR products were visualized on $1.5 \%$ agarose gels (Promega) and analysed using DNA size imaging software (Kodak).

\section{RESULTS}

\section{Screening $M$. avium subsp. paratuberculosis genomic library for IS900 loci}

Two M. avium subsp. paratuberculosis genomic libraries screened for the presence of IS900 yielded 55 positive clones, which were grown to purity and their vector/insert DNA purified. Restriction analysis of these clones identified 14 with a unique sequence in IS900 flanking DNA. These loci were designated numbers 1-14. These loci were then sequenced outwards from the IS900 copy using IS900-specific primers (IS9R and IS9L) and verified in the opposite orientation by resequencing using vector primers (SP6 and T7) and locus-specific primers (LocL and LocR) as described. This generated genomic sequences ranging from 230 to $1171 \mathrm{bp}$ immediately adjacent to the 14 different IS900 insertion loci. The GenBank accession numbers of these genomic sequences are AJ011838, AJ250015-AJ250023 and AJ251434-AJ251437.

\section{BLAST analysis}

BLASTX alignments of sequences of flanking DNA from each IS900 locus made against the M. avium subsp. avium genome (TIGR strain 104) showed 94-100\% homology to corresponding genes (Table 2) for all loci except locus 6. This locus was absent from the M. avium subsp. avium strain 104 genome, but a TBLASTN search with ORFs either side of locus 6 showed homology (38\% identity) to a polyketide synthase of $M$. tuberculosis ( $p k s 17: \mathrm{Rv} 1663$ ) and a transcription regulator (38\% identity) in S. coelicolor (tetR: SCJ11.45c). Loci 4, 6,8 and 11 corresponded to the IS900 insertions previously described as pMB22, pMBJ3, pMBJ2 and pMBL15, respectively (McFadden et al., 1987; Green et al., 1989; Moss et al., 1992; Hernandez Perez et al., 1994). In addition, although minor sequence differences were observed, locus 3 was equivalent to pMB55. Alignment of IS900 loci with homologous M. avium subsp. avium genomic regions showed the target consensus sequence for IS900 insertion to be AAGGAG*A $(\mathrm{N})_{4-7}$ CATG where * indicates the insertion point (Fig. 1). TBLASTN alignments of ORFs predicted within loci 2, 8 and 13 showed no significant homologies with current GenBank accessions. ORFs predicted within loci 1, 3-7, 9-12 and 14 showed $37-88 \%$ identity to $M$. tuberculosis genes (Fig. 2). The orientation, as defined in the Introduction, of IS900 relative to the putative direction of transcription for immediately adjacent ORFs was determined for each locus (Fig. 2). IS900 at loci 7, 9 and 13 were in the hed orientation alone, being inserted into the RBS of the immediately adjacent gene causing a disruption of the existing RBS (consensus AAGGAG) and replacement with an IS900-derived RBS (consensus AAGGAA), positioned two bases closer to the downstream initiation codon. IS900 insertions in loci 4, 8 and 12 disrupted putative ORFs in the hed orientation. There were only two loci (5 and 11) in which IS900 was inserted in the p43 orientation, in each case causing a disruption of the target ORF. At the remaining six loci $(1,2,3,6,10$ and 14), IS900 was inserted in both hed and $p 43$ orientations as defined, in that hed inserted in the target site upstream to its direction of transcription, and $p 43$ did the same in the opposite direction (Fig. 2).

\section{RFLP profiles}

A panel of 81 strains of $M$. avium subsp. paratuberculosis, from 10 different countries, including isolates of bovine, ovine, caprine and human origin (Table 1) were selected and typed by BstEII RFLP, PstI RFLP and MPIL. These 81 strains could be divided using PstI/BstEII RFLP into 17 distinct types (Table 1). In the 
Table 2. Homology and location of M. avium subsp. paratuberculosis IS900 insertion locus sequences in $M$. avium subsp. avium genome

\begin{tabular}{|cccc|}
\hline Locus & $\begin{array}{c}\text { TIGR } \\
\text { clone no. }\end{array}$ & $\begin{array}{c}\text { Position of insertion } \\
\text { in TIGR clone }(\mathbf{b p})\end{array}$ & $\begin{array}{c}\text { Number of bp aligned: } \\
\text { homology }(\%)\end{array}$ \\
\hline 1 & 138 & 4283 & $900: 98$ \\
2 & 31 & 20367 & $1371: 98$ \\
3 & 31 & 8828 & $1007: 98$ \\
4 & 111 & 6013 & $1695: 95$ \\
5 & 45 & 64019 & $432: 100$ \\
6 & - & - & $1707: 0$ \\
7 & 208 & 835 & $600: 99$ \\
8 & 136 & 9109 & $400: 100$ \\
9 & 218 & 7414 & $1108: 98$ \\
10 & 200 & 5449 & $1149: 99$ \\
11 & 24 & 10971 & $449: 94$ \\
12 & 196 & 5684 & $2757: 97$ \\
13 & 31 & 27415 & $1114: 97$ \\
14 & 107 & 348 & $607: 98$ \\
\hline
\end{tabular}

*TIGR clone data obtained from TIGR website at http://www.tigr.org characterizing sites in the sequence of M. avium subsp. avium strain 104 homologous to the insertion loci of IS900 in M. avium subsp. paratuberculosis.

\begin{tabular}{|c|c|c|c|}
\hline & & hed $\longrightarrow$ & \\
\hline cus 1 & CGGCCAACCCAGAAGGAG & ATTCTC - AGGTAAGG & AATTCCGTGTAC \\
\hline cus & CCCCACTTGGGGAAGGGG & ATTCTC - AGGTAAGG & GGCTCATGACC \\
\hline ocus 3 & TCCGACATOCCCAGGG & ATTCTC - AGGTAAGG & AACCACCATGACC \\
\hline Locus 4 & GGAATGTTCGCGAAGGAG & ATTCTC - AGGTAAGG & AACACCACATGACC \\
\hline Locus 5 & TGGCCAATTCCTGGAATG & ATTCTC - AGGTAAGG & ACATACATGACC \\
\hline Locus 6 & TCAATAATCGTCAAGGAG & ATTCTC - AGGTAAGG & ACCACATGTOT \\
\hline Locus 7 & ATCCACAACGCAAAGGAG & ATTCTC - AGGTAAGG & ACAACCATGTAC \\
\hline Locus 8 & ACGGGGAGTOCAAGGAG & ATTCTC - AGGTAAGS & ATCGTCATGGTG \\
\hline Locus 9 & CAGCAGCCATCGAAGGGG & ATTCTC - AGGTAAGG & ACAGCATGATC \\
\hline Locus 10 & CCACGCTOCGACAAGGAG & ATTCTC - AGGTAAGG & ACCGACATGGGA \\
\hline Locus 11 & ACTTTTCCGCGTAAGGGG & ATTCTC - AGGTAAGG & AAACACATGTCG \\
\hline ecus 12 & ATTCCCGGCGCCAAGGAG & ATTCTC - AGGTAAGG & AAACACATGTCG \\
\hline Locus & TTCTCCGACTAAAGGAG & ATTCTC - AGGTAAGG & CCGTGCCATGACT \\
\hline & GCTCGTCGAGAGAAGGAG & ATTCTC - AGGTAAGG & AACATCATGTCC \\
\hline onsensus & AAGGAG & & $* A(N) \ldots$ CATG \\
\hline
\end{tabular}

Fig. 1. Alignment of $\mathrm{IS} 900$ insertions $(*)$ into the $M$. avium subsp. paratuberculosis genome in the hed orientation. Insertion points were determined by comparison with equivalent loci in the $M$. avium subsp. avium (TIGR strain 104) genome.

BstEII RFLP profiles shown in Fig. 3 each band has been allocated a number prefixed by $\mathrm{K}$ indicating its size. In Table 3 , we have taken the B-C1 PstI/BstEII RFLP type as the reference profile and defined other profiles by listing additional or deleted bands using their BstEII Knumber designations.

\section{MPIL analysis and apparent genomic rearrangements around IS900}

Using combinations of locus-specific primers (LocR and LocL) from each side of 14 IS900 insertions, we examined the 17 PstI/BstEII RFLP types and found 10 different MPIL types (M1-M10). These MPIL types correspond to strains of $M$. avium subsp. paratuberculosis which either lack an IS900 element at one or more conserved locus, or which have an apparent genomic rearrangement involving the DNA flanking an IS900 locus. MPIL types M2-M10 matched exactly strains with the PstI/BstEII RFLP types E-C1, B-C5, CS1, F-I1, B-C3, B-C9, K-C11, B-C15 and A-C12, respectively (Table 3 ). Strains of MPIL type M1 contained all 14 loci filled with IS900 and matched strains with 8 different PstI/BstEII RFLP types defined (Fig. 3) by 5 unique $(\mathrm{K} 1 \cdot 4, \mathrm{~K} 1 \cdot 9, \mathrm{~K} 2 \cdot 5, \mathrm{~K} 4 \cdot 1, \mathrm{~K} 3 \cdot 3)$ and 3 shared polymorphisms [K3·1, K3·2(N), K4·0; Table 3]. Further cloning and sequence analysis of these extra BstEII RFLP bands may characterize IS900 insertion loci not described in this study, and may further resolve these $B s t$ EII RFLP types and lead to an improvement in multiplex typing of these strains.

Strains of MPIL type M2 corresponded to the PstI/BstEII RFLP E-C1 profile. They did not amplify a PCR product with a locus-6-spanning primer pair (Loc6R/Loc6L), with MPIL involving Loc6R/IS9L and Loc6L/IS9R primer sets (see Fig. 5, lanes M2), or with primer pairs (pks.L/pks.R and tetR.L/tetR.R) designed to amplify flanking DNA on one or other side of the locus 6 insertion site. Strains of MPIL type M3 corresponded to the PstI/BstEII RFLP B-C5 profile. These strains amplified a product with the Loc5R/Loc5L primer pair, whose size was consistent with that locus being 'unfilled' by IS900. This locus encodes the desA1 gene, which has $84 \%$ identity over 361 bp ( $78 \%$ identity over 116 aa) with desA2 in M. avium subsp. paratuberculosis. Both desA1 and desA2 are $100 \%$ identical to the corresponding genes in M. avium subsp. avium (TIGR strain 104). The close homology between the two genes 
Locus

1

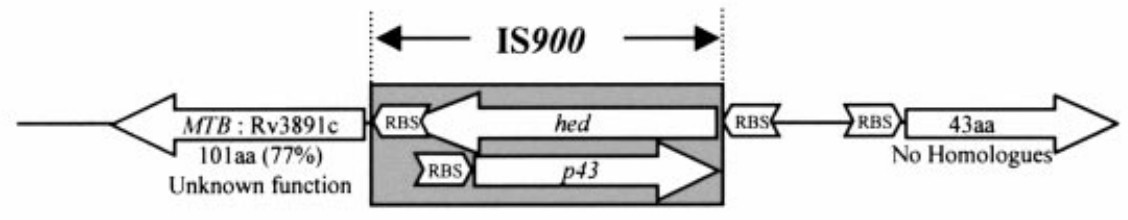

2

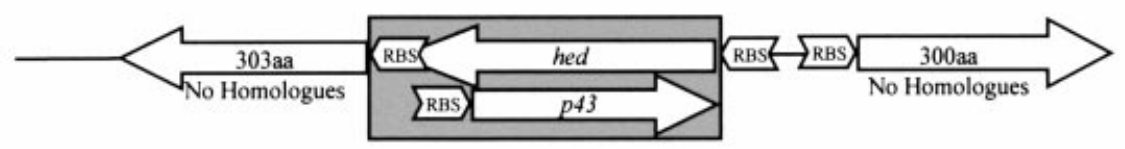

3
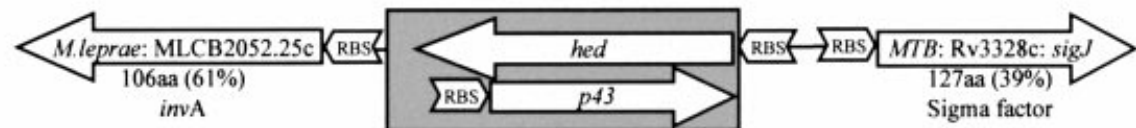

4
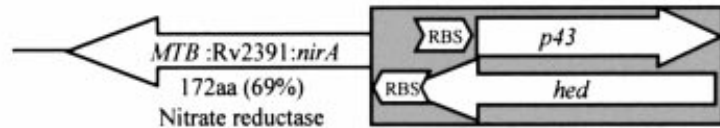

nirA (5aa)

Sigma factor

5

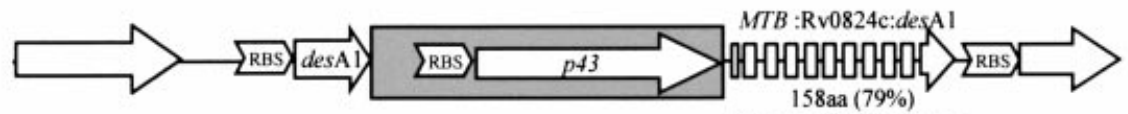

6
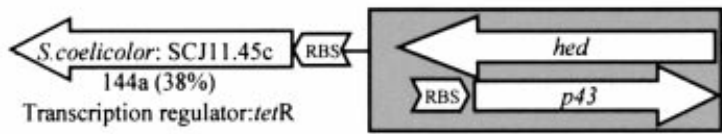

Steroyl-ACP-desaturase

RBS RBS MTB: Rv1663:pks /

57 aa $(38 \%)$
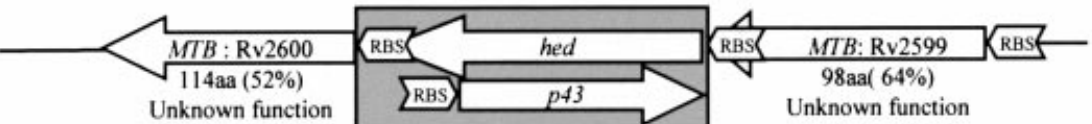
Unknown function Unknown function

8
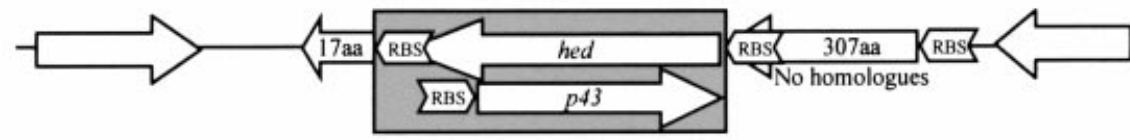
$\sqrt{\text { No homologues }}$

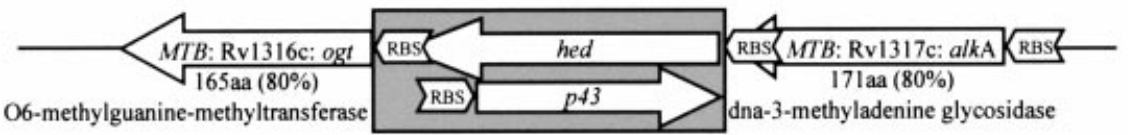

10

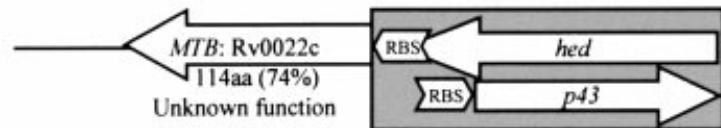
RAS RRS MTB: Rv0023 141 aa $(88 \%)$ Unknown function

11

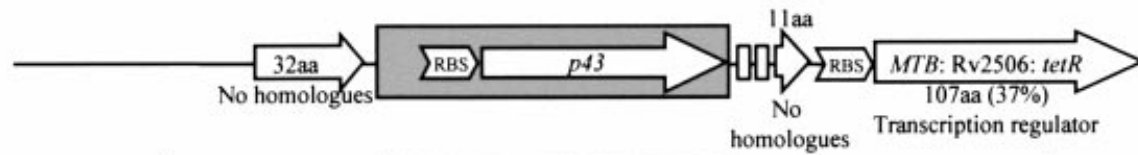

12

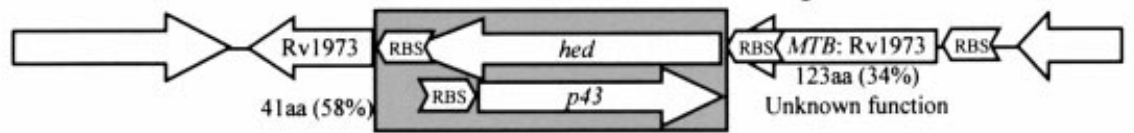

13

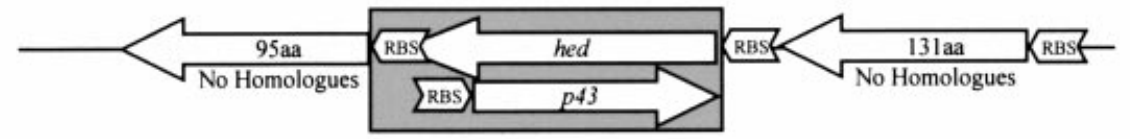

14

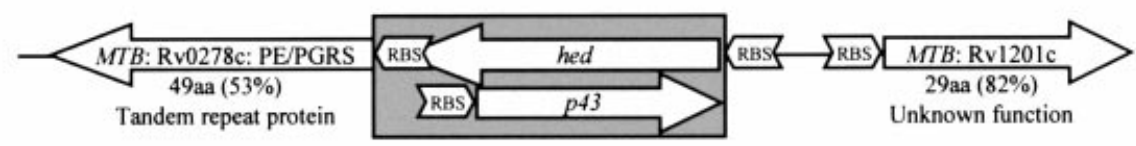

Fig. 2. For legend see facing page. 

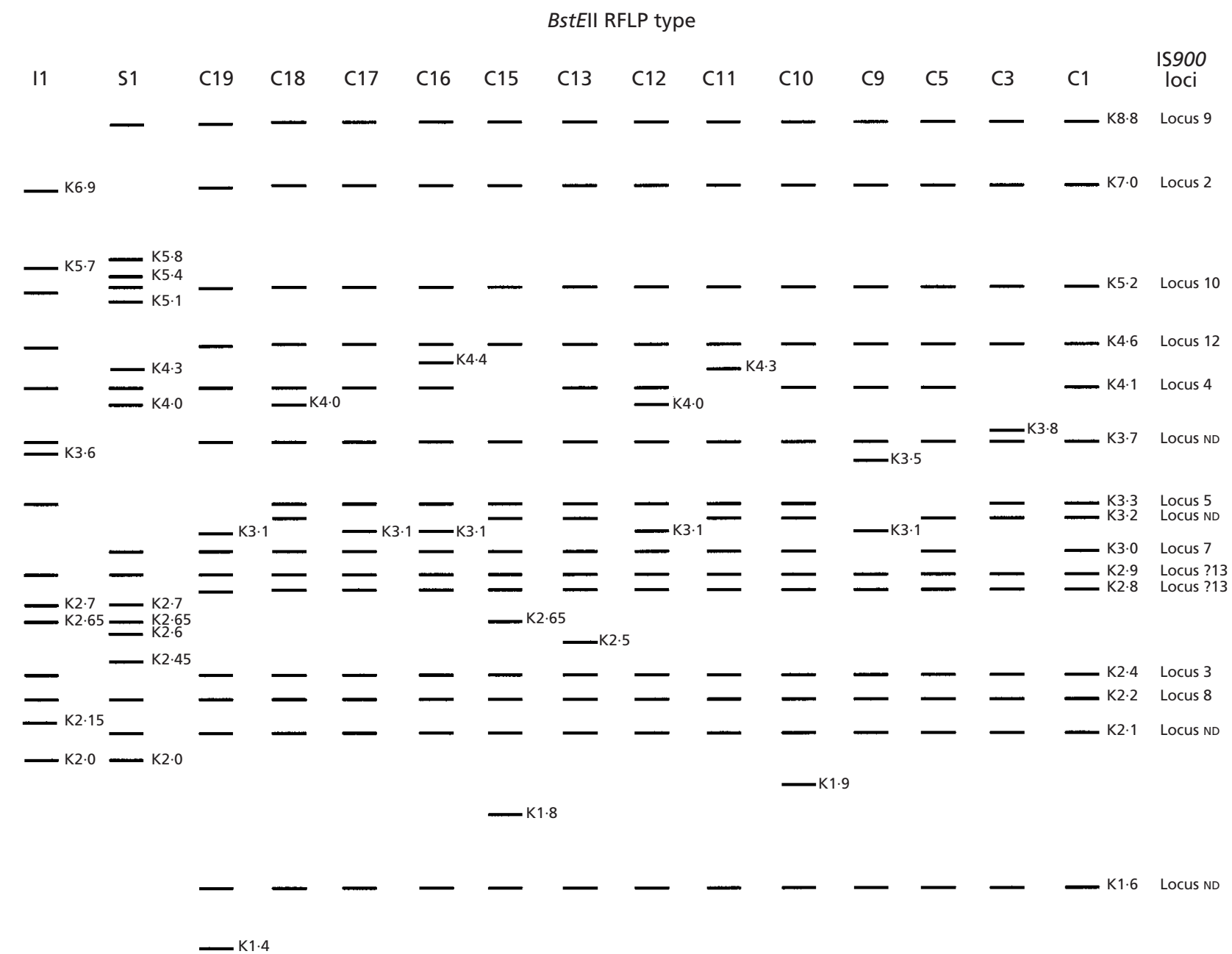

Fig. 3. Diagrammatic presentation of 15 different $M$. avium subsp. paratuberculosis BstEll RFLP profiles obtained by scanning with GelCompar software. C1 is taken as the reference BstEII RFLP profile and individual bands are designated by $\mathrm{K}$ and a number corresponding to the size of each band in kb. The BstEII RFLP profiles C3-C19, S1 and I1 are as previously designated (Pavlik et al., 1999), with the size of new bands differing from the reference profile designated by $\mathrm{K}$ and a number as before. The IS900 locus corresponding to each band is shown where this is known. ND, Could not be determined. 11 and S1 are profiles from sheep strains.

allows non-specific amplification of Loc5R and Loc5L primers with the $\operatorname{des} A 2$ gene. It was not possible therefore to determine if the region encoding desA1 was deleted, or present and not truncated in these strains.

Strains of MPIL type M4 corresponded to the PstI/BstEII RFLP C-S1 profile. These strains amplified 'unfilled' sized products with the Loc $2 \mathrm{R} /$ Loc $2 \mathrm{~L}$ and Loc11R/ Loc11L primer pairs. In addition, they amplified primer pairs Loc6R/IS9L, pks.R/pks.L and tetR.R/tetR.L, but did not amplify Loc6R/Loc6L or Loc6L/IS9R, suggesting a genomic rearrangement involving the tet $R$ side of this locus. Strains of MPIL type M5 corresponded to the PstI/BstEII RFLP F-I1 profile. These strains also

Fig. 2. Diagramatic representation of 14 IS900 loci in M. avium subsp. paratuberculosis. IS900 is represented by shaded boxes. ORFs and predicted direction of transcription are shown by open arrows, and by broken arrows where the reading frame is predicted to be disrupted by the IS900 insertion. The hed arrow has been omitted in loci in which hed is not predicted to be transcribed due to either the absence of a RBS (locus 5) or uncertainty as to the direction of genomic transcription (locus 11). RBS are shown by chevrons. ORFs and RBS in the M. avium subsp. paratuberculosis genome are assigned by reference to homologues and notations in the $M$. tuberculosis (Cole et al., 1998), M. leprae (Eiglmeier et al., 1993) and S. coelicolor (Redenbach et al., 1996) genomes. Loci 1, 2, 4, 7, 8, 9, 10, 12, 13 and 14 have the putative RBS, encoded within I5900, associated with a start codon in the flanking genomic DNA immediately downstream of hed. Putative functions, percentage identity to $M$. avium subsp. paratuberculosis genes and number of TBLASTN aligned residues (aa) are indicated. The length (aa) of ORFs with no current homologues in GenBank are also shown. The figure is not to scale. 
Table 3. Notation and comparison of MPIL and BstEII RFLP profiles

RFLP and MPIL types are as indicated in the text. B-C1 was taken as the reference Pst I/BstEII RFLP profile.

\begin{tabular}{|c|c|c|c|c|}
\hline MPIL type & MPIL profile notation* & $\begin{array}{l}\text { PstI/BstEII } \\
\text { RFLP type }\end{array}$ & BstEII RFLP profile notation $\dagger$ & $\begin{array}{l}\text { No. of } \\
\text { strains }\end{array}$ \\
\hline M1 & Loci 1-14 filled with IS900 & $\begin{array}{l}\text { B-C1 } \\
\text { A-C10 } \\
\text { D-C12 } \\
\text { L-C13 } \\
\text { B-C16 } \\
\text { B-C17 } \\
\text { B-C18 } \\
\text { B-C19 }\end{array}$ & $\begin{array}{l}\text { K } 1 \cdot 9 \\
\mathrm{~K} 4 \cdot 0, \mathrm{~K} 3 \cdot 2(\mathrm{~N}), \mathrm{K} 3 \cdot 1 \\
\mathrm{~K} 2 \cdot 5 \\
\mathrm{~K} 4 \cdot 4, \mathrm{~K} 3 \cdot 2(\mathrm{~N}), \mathrm{K} 3 \cdot 1 \\
\mathrm{~K} 3 \cdot 2(\mathrm{~N}), \mathrm{K} 3 \cdot 1 \\
\mathrm{~K} 4 \cdot 0 \\
\mathrm{~K} 3 \cdot 3(\mathrm{~N}), \mathrm{K} 3 \cdot 2(\mathrm{~N}), \mathrm{K} 3 \cdot 1, \mathrm{~K} 1 \cdot 4\end{array}$ & $\begin{array}{r}32 \\
3 \\
4 \\
3 \\
1 \\
7 \\
1 \\
1\end{array}$ \\
\hline M2 & Locus 6: missing & E-C1 & - & 11 \\
\hline M3 & Locus 5: unfilled & B-C5 & $\mathrm{K} 3 \cdot 3(\mathrm{~N})$ & 6 \\
\hline M4 & $\begin{array}{l}\text { Locus 2: unfilled } \\
\text { Locus 11: unfilled } \\
\text { Locus 6: GR }\end{array}$ & C-S1 & $\begin{array}{l}\mathrm{K} 7 \cdot 0(\mathrm{~N}), \mathrm{K} 5 \cdot 8, \mathrm{~K} 5 \cdot 4, \mathrm{~K} 5 \cdot 1, \\
\mathrm{~K} 4 \cdot 6(\mathrm{~N}), \mathrm{K} 4 \cdot 3, \mathrm{~K} 4 \cdot 0, \mathrm{~K} 3 \cdot 7(\mathrm{~N}), \\
\mathrm{K} 3 \cdot 3(\mathrm{~N}), \mathrm{K} 3 \cdot 2(\mathrm{~N}), \mathrm{K} 2 \cdot 8(\mathrm{~N}), \mathrm{K} 2 \cdot 7, \\
\mathrm{~K} 2 \cdot 65, \mathrm{~K} 2 \cdot 6, \mathrm{~K} 2 \cdot 45, \mathrm{~K} 2 \cdot 4(\mathrm{~N}), \mathrm{K} 2 \cdot 0, \\
\mathrm{~K} 1 \cdot 6(\mathrm{~N})\end{array}$ & 2 \\
\hline M5 & $\begin{array}{l}\text { Locus 2: unfilled } \\
\text { Locus 11: unfilled }\end{array}$ & F-I1 & $\begin{array}{l}\mathrm{K} 7 \cdot 0(\mathrm{~N}), \mathrm{K} 6 \cdot 9, \mathrm{~K} 8 \cdot 8(\mathrm{~N}), \mathrm{K} 5 \cdot 7, \\
\mathrm{~K} 3 \cdot 6, \mathrm{~K} 3 \cdot 2(\mathrm{~N}), \mathrm{K} 3 \cdot 0(\mathrm{~N}), \mathrm{K} 2 \cdot 8(\mathrm{~N}), \mathrm{K} 2 \cdot 7, \\
\mathrm{~K} 2 \cdot 65, \mathrm{~K} 2 \cdot 15, \mathrm{~K} 2 \cdot 1(\mathrm{~N}), \mathrm{K} 2 \cdot 0, \mathrm{~K} 1 \cdot 6(\mathrm{~N})\end{array}$ & 2 \\
\hline M6 & Locus 4 and 7 : IS900GR & $\mathrm{B}-\mathrm{C} 3$ & $\mathrm{~K} 3 \cdot 0(\mathrm{~N}), \mathrm{K} 3 \cdot 8, \mathrm{~K} 4 \cdot 1(\mathrm{~N})$ & 3 \\
\hline M7 & Locus 5 and 7 : IS900GR & B-C9 & $\mathrm{K} 3 \cdot 5, \mathrm{~K} 3 \cdot 3(\mathrm{~N}), \mathrm{K} 3 \cdot 2(\mathrm{~N}), \mathrm{K} 3 \cdot 1, \mathrm{~K} 3 \cdot 0(\mathrm{~N})$ & 1 \\
\hline M8 & $\begin{array}{l}\text { Locus } 4 \text { and } 6: \text { IS900GR } \\
\text { Locus } 12: \text { GR }\end{array}$ & $\mathrm{K}-\mathrm{C} 11$ & $\mathrm{~K} 4 \cdot 3, \mathrm{~K} 4 \cdot 1(\mathrm{~N})$ & 2 \\
\hline M9 & Locus 4 and 6: IS900GR & B-C15 & $\mathrm{K} 4 \cdot 1(\mathrm{~N}), \mathrm{K} 2 \cdot 65, \mathrm{~K} 1 \cdot 8$ & 1 \\
\hline M10 & Locus 12: GR & $\mathrm{A}-\mathrm{C} 12$ & $\mathrm{~K} 4 \cdot 0, \mathrm{~K} 3 \cdot 2(\mathrm{~N}), \mathrm{K} 3 \cdot 1$ & 1 \\
\hline
\end{tabular}

*Unfilled indicates the presence of an insertion site which does not contain an IS900 element. IS900GR indicates an apparent rearrangement involving genomic DNA flanking two separate IS900 loci. GR indicates the situation in which the IS900 element and flanking genomic DNA on one side is present, whilst flanking genomic DNA on the other side of the element is not amplified.

$\dagger(N)$ indicates the absence of a designated band present in the reference profile. New K numbers refer to new bands appearing in these profiles, not present in the reference profile.

amplified 'unfilled' sized products with the Loc2R/ Loc2L and Loc11R/Loc11L primer pairs, but gave 'filled' products for Loc6R/Loc6L. Strains of MPIL type M6 corresponded to the PstI/BstEII RFLP B-C3 profile. These strains did not generate amplification products with locus-4- or locus-7-spanning primer pairs (Loc4L/ Loc4R, Loc7L/Loc7R), but did generate products of expected sizes with primer pairs Loc7R/Loc $4 \mathrm{~L}$ and Loc7L/Loc4R. Overall, these results suggest that a genomic rearrangement may have occurred between locus 4 and locus 7 .

Strains of MPIL type M7 corresponded to the PstI / BstEII RFLP B-C9 profile. These strains did not generate amplification products with locus-5- or locus-7-spanning primer pairs (Loc5L/Loc5R, Loc7L/Loc7R), but did generate products of expected sizes with primer pairs Loc7R/Loc5L and Loc7L/Loc5R. These results suggest that a genomic rearrangement may have occurred between locus 5 and locus 7 (Fig. 4). Strains of
MPIL type M8 corresponded to the PstI/BstEII RFLP KC11 profile. These strains did not generate amplification products with locus-4- or locus-6-spanning primer pairs (Loc4L/Loc4R, Loc6L/Loc6R), but did generate products of expected sizes with primer pairs Loc6R/ Loc $4 \mathrm{~L}$ and Loc6L/Loc4R. These results suggest that a genomic rearrangement may have occurred between locus 6 and locus 4. In addition, these strains gave amplification products with Loc12R/IS9L, but did not amplify the primer pairs Loc12R/Loc12L or Loc12L/ IS9R. This suggests that mutations within the genomic location of primer Loc12L, or a genomic rearrangement involving the Loc12L side of locus 12 with an undetermined locus, has occurred in these strains. Strains of MPIL type M9 corresponded to the PstI/BstEII RFLP B-C15 profile. These strains did not generate amplification products with locus-4- or locus-6-spanning primer pairs (Loc4L/Loc4R, Loc6L/Loc6R), but did generate products of expected sizes with primer pairs Loc6R/Loc4L and Loc6L/Loc4R. These results suggest 
MPIL type M1

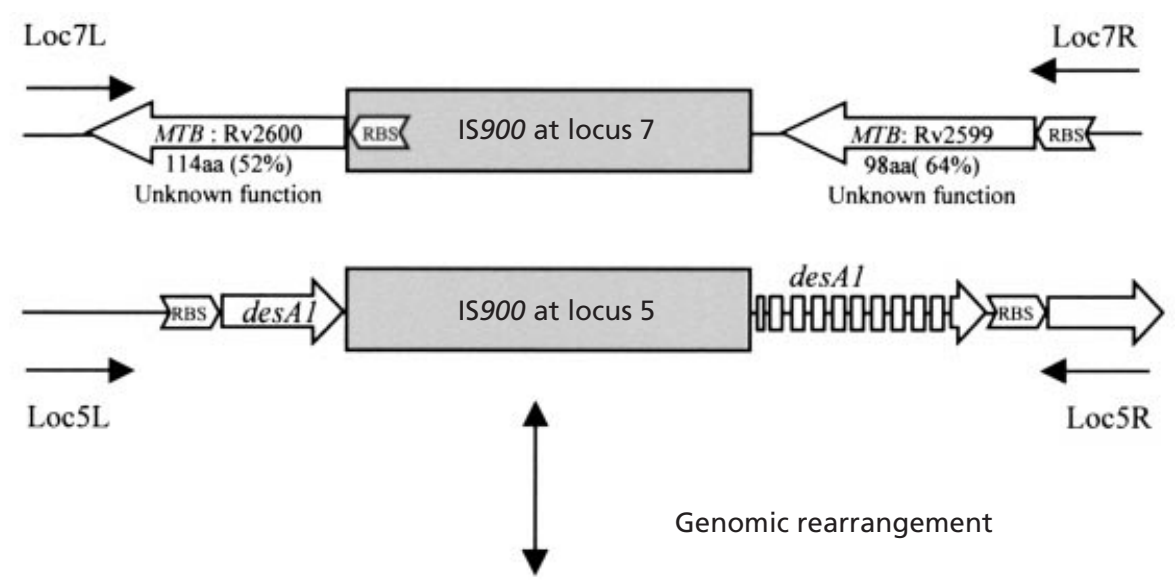

MPIL type M7
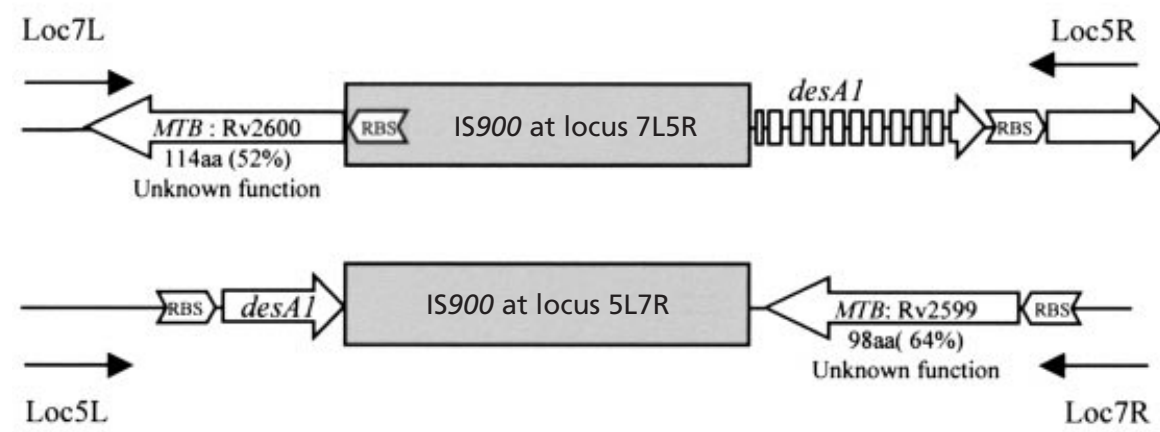

Fig. 4. Proposed genomic rearrangement between locus 5 and locus 7 in $M$. avium subsp. paratuberculosis MPIL type M1 and M7. IS900 is represented by shaded boxes. ORFs are shown by open arrows. ORFs predicted to be disrupted by IS900 are shown by broken arrows. RBS are shown by chevrons. Loc primers used to demonstrate genomic rearrangement are shown by solid arrows.

that a genomic rearrangement may also have occurred in these strains between locus 6 and locus 4. Finally, strains of MPIL type M10 corresponded to the PstI/BstEII RFLP A-C12 profile. These strains gave amplification products with Loc12R/IS9L but did not amplify the primer pairs Loc12R/Loc12L or Loc12L/IS9R as found in MPIL type M8; however these strains differ by containing 'filled' locus 4 and locus 6 sites. MPIL types could be determined, in most cases, using results (Fig. 5) which combined only two multiplex profiles (multiplex 4L1 and multiplex 9R1).

\section{Correlation of RFLP bands with IS900 loci}

The following IS900 loci and BstEII RFLP bands could be correlated (Fig. 3 and Fig. 6 ) : K8·8 = locus 9, K7·0 = locus $2, \mathrm{~K} 5 \cdot 2=$ locus $10, \mathrm{~K} 4 \cdot 6=$ locus $12, \mathrm{~K} 3 \cdot 3=$ locus $5, \mathrm{~K} 3 \cdot 0=$ locus $7, \mathrm{~K} 2 \cdot 4=$ locus $3, \mathrm{~K} 2 \cdot 2=$ locus 8 . The bands K2.8 and K2.9 were too close to separate and therefore either could be attributed to locus 13 . The remaining bands (K3·7, K3·2, K2.1 and K1.6) gave similar strength PCR reaction products from all or none of the fractions and therefore could not be correlated with a particular BstEII RFLP band. The assignment of the K3.3 BstEII RFLP band to IS900 locus 5 is corroborated by the singular absence of K3.3 (Fig. 3) and locus 5 multiplex PCR products (Fig. 5, lane M3) in the PstI/BstEII RFLP type B-C5/MPIL type M3 (Table 3). The assignment of the K3.0 BstEII RFLP band to locus 7 and the K4.1 BstEII RFLP band to locus 4 are corroborated by the absence of both of these bands (Fig. 3, lane C3) and their possible genomic rearrangement (Fig. 4) in the PstI/BstEII RFLP type B-C3/MPIL type M6 (Table 3). Further corroboration of the assignments K3.0 BstEII RFLP band to locus 7 and also the K3.3 BstEII RFLP band to locus 5 is made by the absence of both bands (Fig. 3, lane C9) and their possible genomic rearrangement (Fig. 4) in the PstI/BstEII RFLP type BC9/MPIL type M7 (Table 3).

MPIL and RFLP profiles showed that loci 1, 3, 8, 9, 10, 13 and 14 were conserved in all $81 \mathrm{M}$. avium subsp. paratuberculosis isolates tested. Loci 2, 5, 6 and 11 were present but were 'unfilled' with IS900 in some M. avium subsp. paratuberculosis isolates. Loci 4, 5, 6, 7 and 12 were possibly involved in genomic rearrangements. 

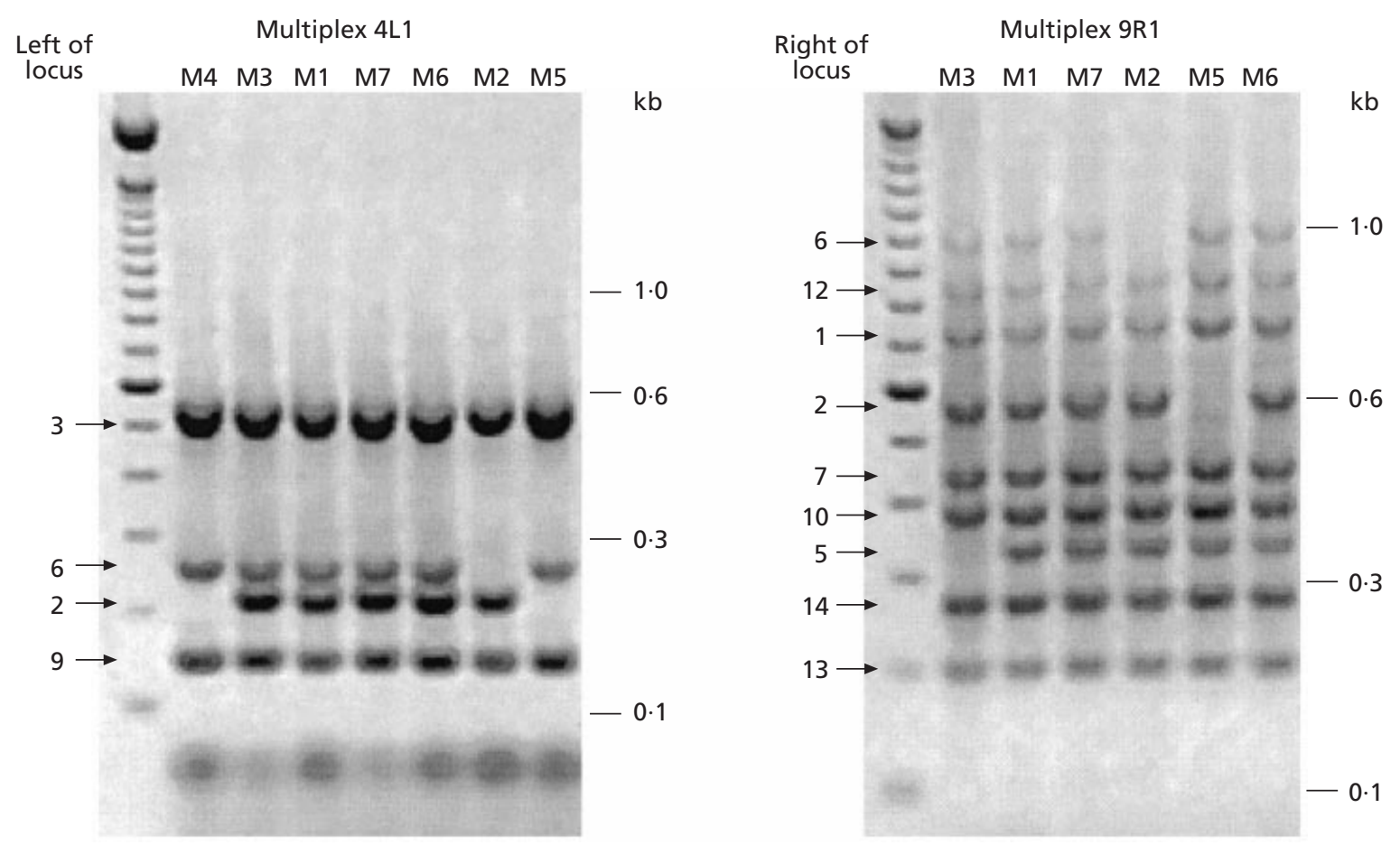

Fig. 5. Agarose gel (1.5\%) electrophoresis of multiplex PCR products. Bands corresponding to specific IS900 insertion loci are shown. Multiplex 4L1 shows the absence of PCR product specific for locus 6 in MPIL type M2 and the absence of PCR product specific for locus 2 in MPIL types M4 and M5. Multiplex 9R1 shows the absence of PCR product specific for the other 'side' of locus 6 in MPIL type M2 and of locus 2 in MPIL type M5, and the absence of specific PCR product for locus 5 in MPIL type M3.

Human isolates exhibited three MPIL types and four PstI/BstEII RFLP types, which corresponded closely to M. avium subsp. paratuberculosis types prevalent in cattle herds.

\section{DISCUSSION}

The present study has characterized genomic DNA and genes flanking 14 IS900 loci in M. avium subsp. paratuberculosis. BLASTX comparisons with the $M$. avium subsp. avium (TIGR strain 104) genome showed that 13 loci were $94-100 \%$ homologous, emphasizing the very close genetic relationship between $M$. avium subsp. paratuberculosis and other organisms of the $M$. avium complex. TBLASTN comparisons of these sequences with GenBank accessions revealed $M$. $t u$ berculosis homologues (37-80\% identity) flanking seven IS900 loci, which could therefore be assigned putative functions (Fig. 2). At four further characterized loci, $M$. tuberculosis homologues (34-88\% identity) although present, could not be assigned a function. Sequences flanking IS900 loci 2, 8 and 13 showed no significant homology to current GenBank accessions on either side of the element.

At loci 4, 8 and 12, IS900 insertion interrupts ORFs transcribed in the hed orientation. At locus 4, this would involve an amino-terminal truncation of an ORF with
$69 \%$ homology to a nitrate reductase (nirA). At loci 5 and 11, the insertion of IS900 interrupts ORFs transcribed in the $p 43$ orientation. In this orientation, the absence of an appropriately positioned RBS encoded by IS900 may result in the disrupted expression of genes downstream of $p 43$. At locus 5 , this involves disruption of an ORF with $79 \%$ homology to an immunodominant cell wall biosynthesis gene, steroyl-ACP desaturase in M. tuberculosis (desA1; Jackson et al., 1997). Sites of IS900 insertion were characterized by the presence of a consensus target sequence AAGGAG*A(N) $)_{4-6}$ CATG (where $*$ denotes the insertion site). IS900 insertion was found to be only in the hed transcriptional orientation relative to this consensus. At seven loci $(1,2,7,9,10,13$ and 14), the IS900 insertion was into the RBS of an ORF disrupting the consensus AAGGAG and replacing it with an IS900 RBS AAGGAA, two bases closer to the downstream initiation codon. Such spatial alterations between RBS and initiation codon have been shown to affect the expression of downstream genes in other genera (Vellanoweth \& Rabinowitz, 1992; Chen et al., 1994). The genes in M. avium subsp. paratuberculosis affected by this substitution, where they are known, include $O-6$-methylguanine methyltransferase $(o g t)$ at locus 9 and a PE/PGRS tandem repeat protein at locus 14. The function of PE/PGRS has been closely associated with antigenic variation in M. tuberculosis (Cole et al., 1998), whilst ogt is a major component of the adaptive 


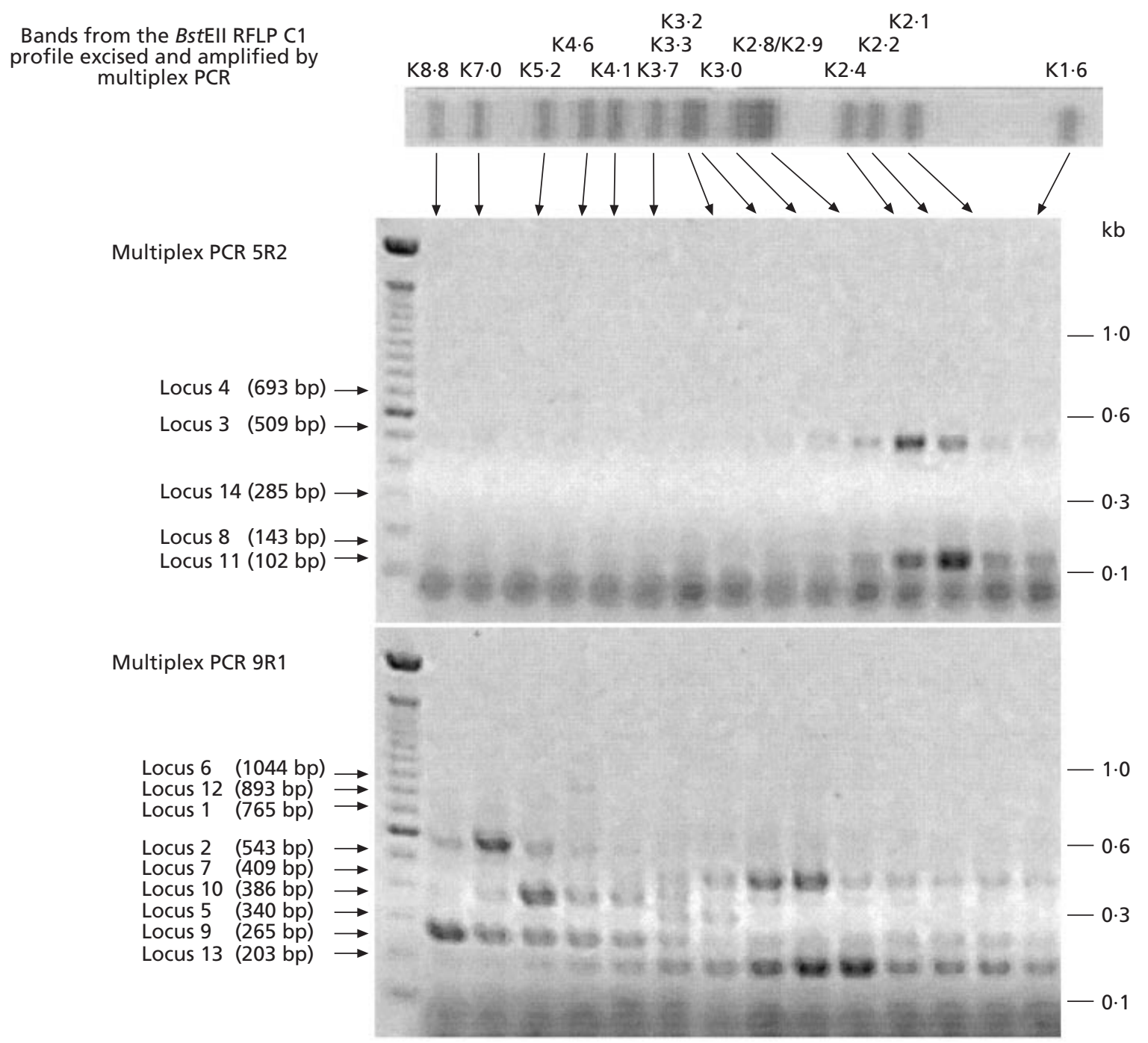

Fig. 6. Multiplex PCR applied to individual bands excised from an IS900 RFLP profile. The top panel shows the BstEII RFLP C1 profile with each of the 15 loci containing 15900 designated according to size as in Table 3 . The middle panel shows the amplification products and their expected sizes resulting from multiplex PCR 5R2 (see Methods) applied separately to each excisable band (K2.8/K2.9 form a single fraction). The lower panel shows the amplification products and their expected sizes resulting from multiplex PCR 9R1 applied to each band/fraction in a similar manner. Loci which could be assigned to individual RFLP bands in these studies were: K8.8 = locus 9, K7.0 = locus 2, K5.2 = locus 10 , K4.6 (weakly shown) $=$ locus $12, \mathrm{~K} 3 \cdot 3=$ locus $5, \mathrm{~K} 3 \cdot 0=$ locus $7, \mathrm{~K} 2 \cdot 4=$ locus $3, \mathrm{~K} 2 \cdot 2=$ locus 8 . Either K2.8 or K2.9 correspond to locus 13. K3.7, K2.1 and $\mathrm{K} 1 \cdot 6$ could not be assigned and $\mathrm{K} 4 \cdot 1$, though not assigned on the present gel, is shown by other data to correspond with locus 4 (see text).

response to intracellular killing in mycobacteria (Lindahl et al., 1988). The presence of an IS900 element may therefore influence the expression of neighbouring genes by substitution and alteration in the spatial orientation of the RBS, by direct disruption of the reading frame, and also by the presence of an IS900encoded promoter controlling $p 43$. Genes immediately flanking IS900 elements are shown here to have putative functions including cell wall antigens, transcription regulators and sigma factors. The differential expression of these important antigens and gene regulators, due to the presence of IS900, could have a significant effect on determining the phenotype of the organism.
A MPIL typing system using PCR primers specific for each IS900 locus was developed to investigate a panel of 81 M. avium subsp. paratuberculosis strains. This found that IS900 insertions into 7 of the 14 loci were conserved in all strains tested, including bovine, ovine, caprine and human isolates. Loci 4, 5 and 7 were also present in all strains, but were involved in apparent genomic rearrangements in some strains which included cross-over of either locus 4 with locus 6 , locus 4 with locus 7 , or locus 5 with locus 7 . We were able to assign 9 of 15 RFLP bands from the reference Pst $\mathrm{I}$ BstEII B-C1 profile to individual sequenced loci. Of the 17 different PstI/BstEII RFLP types, MPIL typing was able to differentiate 10 
MPIL types. In this study, each of nine of these MPIL types corresponded precisely to one distinct PstI/BstEII RFLP type, supporting the validity of the MPIL procedure, and the conclusion that the two typing methods address the same genetic variations. This suggests that MPIL typing may substitute for RFLP typing of these strains, with the advantage, of particular relevance to M. avium subsp. paratuberculosis, of being rapid, and may be applicable to direct typing from a sample without the need for culture (Hermon-Taylor et al., 2000). The remaining MPIL type, M1, corresponded with eight Pst / BstEII RFLP types. Further resolution of this cluster may be possible when the known remaining four IS900 loci are similarly characterized, and the apparent genomic rearrangements involving PstI/BstEII RFLP bands $\mathrm{K} 1 \cdot 4, \mathrm{~K} 1 \cdot 9, \mathrm{~K} 2 \cdot 5, \mathrm{~K} 4 \cdot 0, \mathrm{~K} 4 \cdot 4$ (Table 3) are elucidated. At the present state of development the resolving power of MPIL is less than RFLP.

Previous RFLP typing has shown substantial differences between bovine and ovine strains of $M$. avium subsp. paratuberculosis (Collins et al., 1990; Whipple et al., 1990; Thoresen \& Olsaker, 1994; Pavlik et al., 1995; Bauerfeind et al., 1996). We have shown that in bovine and other strains, locus 2 and locus 11 are consistently occupied by an IS900 element. By contrast, in ovine strains identified by MPIL as M4 and M5, locus 2 and locus 11, although present, appear to be consistently unfilled by an IS900 element. BLAST analysis of these genomic regions in ovine strains of $M$. avium subsp. paratuberculosis shows that they closely resemble the corresponding regions in the M. avium subsp. avium genome and include a homologue to a tet $R$ regulation protein from M. tuberculosis. The relatively high rate of growth of all $M$. avium subsp. avium strains, however, suggests that a differential transcriptomic profile caused by insertions of IS900 at these loci would be unlikely to be related to the very slow-growing phenotype of ovine M. avium subsp. paratuberculosis strains.

An interesting and unexpected finding of this investigation was the absence of locus 6 (MPIL type M2) in 11 of the 81 strains of $M$. avium subsp. paratuberculosis we studied. These 11 isolates were all derived from one herd of cattle imported into the Slovak Republic from Denmark in 1970. Locus 6 was also absent from all seven reference strains of $M$. avium subsp. avium, from one strain of M. avium subsp. silvaticum, as well as from $M$. intracellulare, M. malmoense, M. tuberculosis H37Rv, M. phlei, M. fortuitum and M. smegmatis. The deleted region included the locus 6 flanking genes consisting of a homologue (38\% identity) to the polyketide synthase pks17: Rv1663 of M. tuberculosis (Quadri et al., 1998), and a homologue (38\% identity) of a transcriptional regulator, tetR, from S. coelicolor (Marvaud et al., 1998). The loss of this locus, however, is inconsistent with both MPIL M1 and M4 profiles exhibiting similar BstEII RFLP C1 profiles (Table 3). Locus 6 could not be assigned to a particular RFLP band and it is possible that MPIL M1 type strains have an additional RFLP band that is hidden by the RFLP gel resolution. Further studies on this locus are in progress.

\section{ACKNOWLEDGEMENTS}

This work was supported by grants from The Wellcome Trust and the Ileostomy Association, to whom we express our appreciation.

\section{REFERENCES}

Bartlett, D. H. \& Silverman, M. (1989). Nucleotide sequence of IS492, a novel insertion sequence causing variation in extracellular polysaccharide production in the marine bacterium Pseudomonas atlantica. J Bacteriol 17, 1763-1766.

Bauerfeind, R., Benazzi, S., Weiss, R., Schliesser, T., Willems, H. \& Baljer, G. (1996). Molecular characterization of Mycobacterium paratuberculosis isolates from sheep, goats, and cattle by hybridization with a DNA probe to insertion element IS900. J Clin Microbiol 34, 1617-1621.

Chen, H., Bjerknes, M., Kumar, R. \& Jay, E. (1994). Determination of the optimal aligned spacing between the Shine-Delgarno sequence and the translation initiation codon of Escherichia coli mRNAs. Nucleic Acids Res 25, 4953-4957.

Chiodini, R. J. (1989). Crohn's disease and the mycobacterioses: a review and comparison of two disease entities. Clin Microbiol Rev 2, 90-117.

Cocito, C., Gilot, P., Coene, M., de Kesel, M., Poupart, P. \& Vannuffel, P. (1994). Paratuberculosis. Clin Microbiol Revs 7, 328-345.

Cole, S. T., Brosch, R., Parkhill, J. \& 39 other authors (1998). Deciphering the biology of Mycobacterium tuberculosis from the complete genome sequence. Nature 393, 537-544.

Collins, D. M., Gabric, D. M. \& de Lisle, G. W. (1990). Identification of two groups of Mycobacterium paratuberculosis strains by restriction endonuclease analysis and DNA hybridization. J Clin Microbiol 28, 1591-1596.

Collins, D. M., Cavaignac, S. \& de Lisle, G. W. (1997). Use of four DNA insertion sequences to characterize strains of the $\mathrm{Myco-}$ bacterium avium complex isolated from animals. Mol Cell Probes 11, 373-380.

Doran, T. J., Davies, J. K., Radford, A. J. \& Hodgson, A. L. (1994). Putative functional domain within ORF2 on the Mycobacterium insertion sequences IS900 and IS902. Immunol Cell Biol 72, $427-434$.

Doran, T., Tizard, M., Millar, D., Ford, J., Sumar, N., Loughlin, M. \& Hermon-Taylor, J. (1997). IS900 targets translation initiation signals in Mycobacterium avium subsp. paratuberculosis to facilitate expression of its hed gene. Microbiology 143, 547-552.

Eiglmeier, K., Honore, N., Woods, S. A., Caudron, B. \& Cole, S. T. (1993). Use of an ordered cosmid library to deduce the genomic organization of Mycobacterium leprae. Mol Microbiol 7, 197-206.

El-Zaatari, F. A. K., Naser, S., Engstraud, L., Hachem, C. Y. \& Graham, D. Y. (1994). Identification and characterisation of Mycobacterium paratuberculosis recombinant clones expressed in E. coli. Curr Microbiol 29, 177-184.

Green, E. P., Tizard, M. L. V., Moss, M. T., Thompson, J., Winterbourne, D. J., McFadden, J. J. \& Hermon-Taylor, J. (1989). Sequence and characteristics of IS900, an insertion element identified in a human Crohn's disease isolate of Mycobacterium paratuberculosis. Nucleic Acids Res 17, 9063-9073.

Hermon-Taylor, J., Bull, T. J., Sheridan, J. M., Cheng, J., Stellakis, M. L. \& Sumar, N. (2000). The causation of Crohn's disease by Mycobacterium avium subsp. paratuberculosis. Can J Gastroenterol 14, 521-539. 
Hernandez Perez, M., Fomukong, N. G., Hellyer, T., Brown, I. N. \& Dale, J. W. (1994). Characterization of IS1110, a highly mobile genetic element from Mycobacterium avium. Mol Microbiol 12, 717-724.

Jackson, M., Portnoi, D., Catheline, D., Dumail, L., Rauzier, J., Legrand, P. \& Gicquel, B. (1997). Mycobacterium tuberculosis Des protein: an immunodominant target for the humoral response of tuberculosis patients. Infect Immunol 65, 2883-2889.

Kallastu, A., Horak, R. \& Kivisaar, M. (1998). Identification and characterization of IS1411, a new insertion sequence which causes transcriptional activation of the phenol degradation genes in Pseudomonas putida. J Bacteriol 180, 5306-5312.

Kallinowski, F., Wassmer, A., Hofmann, M. A., Harmsen, D., Heesemann, J., Karch, H., Herfarth, C. \& Buhr, H. J. (1998). Prevalence of enteropathogenic bacteria in surgically treated chronic inflammatory bowel disease. Hepatogastroenterology 45, 1552-1558.

Larsen, A. B., Harley, M. S., Moon, W. \& Merkal, R. S. (1971). Susceptibility of swine to Mycobacterium paratuberculosis. Am J Vet Res 32, 589-595.

Lindahl, T., Sedgewick, B. \& Nakabeppu, Y. (1988). Regulation and expression of the adaptive response to alkylating agents. Annu Rev Biochem 57, 133-157.

McClure, H. M., Chiodini, R. J., Anderson, D. C., Swenson, R. B., Thayer, W. R. \& Coutu, J. A (1987). Mycobacterium paratuberculosis infection in a colony of Stumptail Macaques (Macaca arctoides). J Infect Dis 155, 1011-1019.

McFadden, J. J., Butcher, P. D., Chiodini, R. \& Hermon-Taylor, J. (1987). Crohn's disease isolated mycobacteria are identical to Mycobacterium paratuberculosis, as determined by DNA probes that distinguish between mycobacterial species. J Clin Microbiol 25, 796-801.

Marvaud, J. C., Eisel, U., Binz, T., Niemann, H. \& Popoff, M. R. (1998). TetR is a positive regulator of the tetanus toxin gene in Clostridium tetani and is homologous to botR. Infect Immun 66, 5698-5702.

Moreira, A. R., Paolicchi, F., Morsella, C. \& 7 other authors (1999). Distribution of IS900 restriction fragment length polymorphism types among animal Mycobacterium avium subsp. paratuberculosis isolates from Argentina and Europe. Vet Microbiol 70, 251-259.

Moss, M. T., Green, E. P., Tizard, M. L., Malik, Z. P. \& HermonTaylor, J. (1991). Specific detection of Mycobacterium paratuberculosis by DNA hybridisation with a fragment of the insertion element IS900. Gut 32, 395-398.

Moss, M. T., Malik, Z. P., Tizard, M. L. V., Green, E. P., Sanderson, J. D. \& Hermon-Taylor, J. (1992). IS902, an insertion element of the chronic-enteritis-causing Mycobacterium avium subsp. silvaticum. J Gen Microbiol 138, 139-145.

Pavlik, I., Bejckova, L., Pavlas, M., Rozsypalova, Z. \& Koskova, S. (1995). Characterization by restriction endonuclease analysis and DNA hybridization using IS900 of bovine, ovine, caprine and human dependent strains of Mycobacterium paratuberculosis isolated in various localities. Vet Microbiol 45, 311-318.

Pavlik, I., Horvathova, A., Dvorska, L., Bartl, J., Svastova, P., du Maine, R. \& Rychlik, I. (1999). Standardisation of restriction fragment length polymorphism method for Mycobacterium avium subsp. paratuberculosis. J Microbiol Methods 38, 155-167.

Portaels, F., Larsson, L. \& Smeets, P. (1988). Isolation of mycobacteria from healthy persons' stools. Int J Lepr Other Mycobact Dis 56, 468-471.

Quadri, L. E., Sello, J., Keating, T. A., Weinreb, P. H. \& Walsh, C. T. (1998). Identification of a Mycobacterium tuberculosis gene cluster encoding the biosynthetic enzymes for assembly of the virulence-conferring siderophore mycobactin. Chem Biol 5, 631-645.

Redenbach, M., Kieser, H. M., Denapaite, D., Eichner, A., Cullum, J., Kinashi, H. \& Hopwood, D. A. (1996). A set of ordered cosmids and a detailed genetic and physical map for the $8 \mathrm{Mb}$ Streptomyces coelicolor A3(2) chromosome. Mol Microbiol 21, 77-96.

Thoresen, O. F. \& Olsaker, I. (1994). Distribution and hybridization patterns of the insertion element IS900 in clinical isolates of Mycobacterium paratuberculosis. Vet Microbiol 40, 293-303.

Tizard, M. L., Moss, M. T., Sanderson, J. D., Austen, B. M. \& Hermon-Taylor, J. (1992). p43, the protein product of the atypical insertion sequence IS900, is expressed in Mycobacterium paratuberculosis. J Gen Microbiol 138, 1729-1736.

USDA (1974). Laboratory Methods in Veterinary Mycobacteriology, rev. edn. Ames, IA: Veterinary Services Laboratories.

Vellanoweth, R. L. \& Rabinowitz, J. C. (1992). The influence of ribosome-binding-site elements on translational efficiency in Bacillus subtilis and Escherichia coli in vivo. Mol Microbiol 6, 1105-1114.

Whipple, D., Kapke, P. \& Vary, C. (1990). Identification of restriction fragment length polymorphisms in DNA from $\mathrm{Myco-}$ bacterium paratuberculosis. J Clin Microbiol 28, 2561-2564.

Whittington, R. J., Marsh, I., Turner, M. J., McAllister, S., Choy, E., Eamens, G. J., Marshall, D. J. \& Ottaway, S. (1998). Rapid detection of Mycobacterium paratuberculosis in clinical samples from ruminants and in spiked environmental samples by modified BACTEC 12B radiometric culture and direct confirmation by IS900 PCR. J Clin Microbiol 36, 701-707.

Ziebuhr, W., Krimmer, V., Rachid, S., Lossner, I., Gotz, F. \& Hacker, J. (1999). A novel mechanism of phase variation of virulence in Staphylococcus epidermidis: evidence for control of the polysaccharide intercellular adhesin synthesis by alternating insertion and excision of the insertion sequence element IS256. Mol Microbiol 32, 345-356.

Received 9 December 1999; revised 22 May 2000; accepted 19 June 2000. 